\title{
Obituary
}

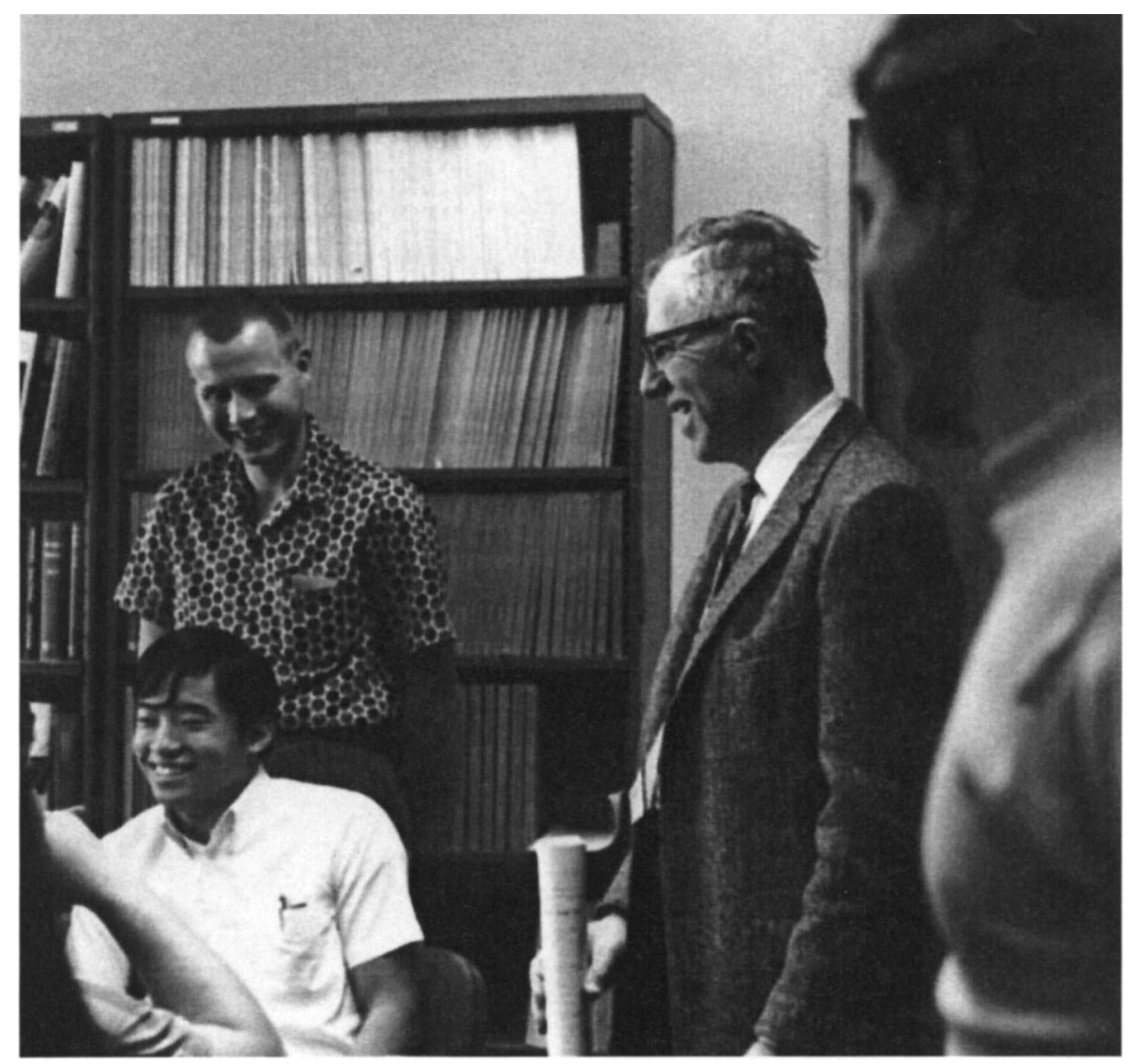

\section{Kenneth Nyitray Trueblood 1920-1998}

Kenneth N. Trueblood, a pioneer in the development of the use of computers for the determination and refinement of crystal structures and in the analysis of molecular motion in the solid state, died of a melanoma at home in Los Angeles on 7 May 1998. He was a much respected scientist and teacher. He leaves a wife, Jean, a graduate of the School of Social Work at Columbia University.

Born in Dobbs Ferry, New York, USA, on 24 April 1920, the youngest of three boys, Ken obtained an AB from Harvard in 1941 and a $\mathrm{PhD}$ in chemistry from Caltech in 1947. After 2 years as a postdoc at Caltech, where he was inspired by the work of Linus Pauling, Ken went to UCLA as an instructor in the Chemistry Department. Within a year he had been made an Assistant Professor, becoming a full Professor in 1960. $\mathrm{He}$ received a Fulbright Award to study at Oxford
University between 1956 and 1957, and he used this occasion to work on vitamin $\mathrm{B}_{12}$ with Dorothy Hodgkin. He was an exchange visitor at the Institute of ElementoOrganic Compounds in Moscow in 1965, where he got to know Kitaigorodsky, and a Visiting Professor at the University of Ibadan, Nigeria, 1964-1965. He also worked with Jack Dunitz when he was on sabbatical at the Swiss Federal Institute of Technology in Zurich, 1976-1977. Ken retired from UCLA in 1989 as Professor Emeritus, but continued with research projects. A small meeting held at that time in his honor demonstrated the affection that his former students and his colleagues had for him. Ken served the crystallographic community as President of the American Crystallographic Association in 1961, member of the US National Committee for Crystallography and its Vice Chair in 1963-1965. He also served with distinction in several administrative posi- 
tions at UCLA - Chair of the Chemistry Department 1965-1970 and 1990-1991 (called back after his retirement), Dean of the UCLA College of Letters and Science from 1971 to 1974, and Chair of the UCLA Academic Senate 1983-1984. In addition to science he loved sports, especially the New York Yankees, and attended as many UCLA games as possible with his wife, Jeanie.

Among the earliest papers from the US on crystallographic computer programs was a gem from Ken's laboratory [Acta Cryst. (1953), 6, 427] on three-dimensional summations on a digital computer. This came at a time when few crystallographers were working with three-dimensional data because the calculations involved were unbelievably tedious and timeconsuming. The US National Bureau of Standards Western Automatic Computer had been installed at UCLA in the early 1950's and Ken set about using it for crystallographic work. The development of suitable programs for calculating electron-density maps and, later, for refining structures by Ken and his students Stan Mayer, Dick Prosen, F. Kruse and Bob Sparks led to his collaboration in 1954 with Dorothy Hodgkin on the crystal structure of vitamin $\mathrm{B}_{12}$. This was the largest unknown chemical structure determined at that time and its solution contributed to the Nobel Prize won by Dorothy. In a collaboration carried out by normal mail between Oxford, England, and California, USA, Ken calculated electron-density maps phased on successively larger and larger portions of the molecule. Ken's part in this story was essential to the determination of the chemical formula of the vitamin [Nature, (1955), 176, 325]. He then continued with the refinement of the structure. Crystallographic computer programs were, at that time, among the most complex written and, because of the amount of memory they used and the amount of time they took, they usually were run at night. The programmer also had to be the computer operator and needed to know how to test and replace tubes, diodes and other portions of the massive machine in addition to rectifying problems in the program. Later Ken and Robert Long developed some of the very first programs for use in direct methods of structure determination.

Ken continued with crystallographic studies of strained polycyclic molecules and host-guest complexes, and also, in collaboration with Verner Schomaker and Jack Dunitz, worked on thermal motion and disorder in the crystalline state and its adequate description (rigidbody analysis, force constants and rotation barriers) [ $J$. Phys. Chem. (1988), 92, 856]. When he was told that his time was limited because of his illness, his major task (which he completed) was to finish a paper with Verner Schomaker (who died in 1997). Uniquely, this final paper immediately follows this obituary. Crystal structures determined by him include those of hydroxyproline and some derivatives of nucleic acid bases, which led to some observations on hydrogen bonding between base pairs with Jerry Donohue. He also studied the structures of some strained organic molecules including hexahelicene, for which he established the absolute configuration, and some cyclophanes with Emily Maverick, Joel Bernstein, Hakon Hope and Carolyn Knobler. Later he studied host-guest complexes with Don Cram, Emily Maverick and Carolyn Knobler.

Ken excelled as a teacher. He was a member of the IUCr Commission on Crystallographic Teaching and obtained the first UCLA Distinguished Teaching Award in 1961, an Award for Excellence in Teaching from the Manufacturing Chemists Association in 1978, a Letters and Science Faculty Award from UCLA in 1982 and the Fankuchen Memorial Award of the American Crystallographic Association in 1995. He taught in many ACAand IUCr-sponsored schools and always provided the students with clear and relevant presentations. His significant contributions to crystallographic teaching were remembered with respect at the opening of the 1998 Erice Crystallography School. In addition, he coauthored Crystal Structure Analysis: A Primer and Chem One, and both texts have influenced several generations of students. At a memorial session for him at the ACA meeting in Washington, July 1998, some of his former students described how he knew the names of each individual member of his classes (no matter how large), demanded high standards from them in experimental work and writing, and was always available for them to talk to. Ken could be gentle but firm, but it was always clear that he wanted to encourage students to do their best and develop their interests and understanding of chemistry. A modest man, he had a fine sense of humor and an infectious chuckle. This enthusiastic pioneer of modern crystallography will be sadly missed.

JENNY P. GLUSKER 